\title{
$\eta$-EINSTEIN SASAKIAN IMMERSIONS IN NON-COMPACT SASAKIAN SPACE FORMS
}

\author{
GIANLUCA BANDE, BENIAMINO CAPPELLETTI-MONTANO, AND ANDREA LOI
}

\begin{abstract}
The aim of this paper is to study Sasakian immersions of (non-compact) complete regular Sasakian manifolds into the Heisenberg group and into $\mathbb{B}^{N} \times \mathbb{R}$ equipped with their standard Sasakian structures. We obtain a complete classification of such manifolds in the $\eta$-Einstein case.
\end{abstract}

\section{INTRODUCTION}

Sasakian geometry is considered as the odd-dimensional counterpart of Kähler geometry. Despite the Kähler case, where the study of Kähler immersions is well developed, due to the seminal work of Calabi [6] (see also [15] for a modern treatment and an account on the subject), in the Sasakian setting there are few results. Most of the Sasakian results are concerned with finding conditions which ensure that a Sasakian submanifold is totally geodesic or similar geometric properties (see, for instance, $[12,13,14]$ ).

In [7] the second and the third authors studied Sasakian immersions into spheres. In particular they proved the following classification result:

Theorem ([7]). Let $S$ be a $(2 n+1)$-dimensional compact $\eta$-Einstein Sasakian manifold. Assume that there exists a Sasakian immersion of $S$ into $\mathbb{S}^{2 N+1}$. If $N=n+2$ then $S$ is Sasaki equivalent to $\mathbb{S}^{2 n+1}$ or to the Boothby-Wang fibration over $Q_{n}$, where $Q_{n} \subset \mathbb{C} P^{n+1}$ is the complex quadric equipped with the restriction of the Fubini-Study form of $\mathbb{C} P^{n+1}$.

Since the (Sasakian) sphere is one of the three "models" of Sasakian space forms, it is quite natural to study as a second step the immersions into Sasakian space forms.

In this paper we give a complete characterisation of Sasakian immersions of complete, regular, $\eta$-Einstein Sasakian manifolds into a non-compact Sasakian space form $M(N, c)$, proving the following:

THEOREM 1. Let $S$ be a $(2 n+1)$-dimensional connected, complete, regular $\eta$-Einstein Sasakian manifold. Suppose that there exists $p \in S$, an open neightborhood $U_{p}$ of $p$ and a Sasakian immersion $\phi: U_{p} \rightarrow M(N, c)$, where $c \leq-3$. Then $S$ is Sasaki equivalent to $M(n, c) / \Gamma$ where $\Gamma$ is some discrete subgroup of the Sasakian-isometry group of $M(n, c)$. Moreover, if $U_{p}=S$ then $\Gamma=\{1\}$ and $\phi$ is, up to a Sasakian transformation of $M(N, c)$, given by

$$
\phi(z, t)=(z, 0, t+c)
$$

Date: February 17, 2020.

2010 Mathematics Subject Classification. 53C25; 53C55.

Key words and phrases. Sasakian; Sasaki-Einstein; $\eta$-Einstein; Sasakian immersion; Kähler manifolds; Kähler immersions, Sasakian space form.

The authors were supported by Prin 2015 - Real and Complex Manifolds: Geometry, Topology and Harmonic Analysis - Italy and by Fondazione di Sardegna (Project STAGE) and Regione Autonoma della Sardegna (Project KASBA). All the authors are members of INdAM-GNSAGA - Gruppo Nazionale per le Strutture Algebriche, Geometriche e le loro Applicazioni. 
Theorem 1 is a strong generalisation of [12, Theorem 3.2] which asserts that a complete, $\phi$-invariant, $\eta$-Einstein submanifold of codimension 2 of the $(2 N+1)$-dimensional Heisenberg group is necessarily a totally geodesic submanifold Sasaki-equivalent to a copy of a $(2 N-1)$-dimensional Heisenberg group and similarly for totally geodesic submanifolds of $\mathbb{B}^{N} \times \mathbb{R}$, where $\mathbb{B}^{N}$ denotes the unit disc of $\mathbb{C}^{N}$ equipped with the hyperbolic metric. In fact in our result there is no restriction on the codimension and we assume that we have a Sasakian immersion instead of a $\phi$-invariant submanifold. Moreover the immersion is not necessarily injective and is not assumed to be from the whole space but from an open neighbourhood of a point.

The general philosophy in [7] and in this paper is to take into account the transversal Kähler geometry of the Reeb foliation. When a regular Sasakian manifold is compact as in [7], one can use the so-called Boothby-Wang construction [3], which realises the space of leaves as a Kähler manifold which is the base of a principal $\mathbb{S}^{1}$-fibration. Then one translates the immersion problem into a Kähler immersion problem of the base spaces.

Trying the same trick in the non-compact case is more complicated because the BoothbyWang construction fails in general, even if the Sasakian manifold is regular. Nevertheless, the Reeb foliation has the strong property to be both a totally geodesic and a Riemannian foliation. Assuming the Sasakian manifold complete, one can appeal to the result of Reinhart [19] which says that the space of leaves is the base space of a fibration, and once again translate the problem into one on Kähler immersions.

The paper contains two other sections. In Section 2 we recall the main definitions and some foliation theory needed in the proof of Theorem 1 to whom Section 3 is dedicated.

\section{Preliminaries}

A contact metric manifold is a contact manifold $(S, \eta)$ admitting a Riemannian metric $g$ compatible with the contact structure, in the sense that, defined the $(1,1)$-tensor $\phi$ by $d \eta=2 g(\cdot, \phi \cdot)$, the following conditions are fulfilled

$$
\phi^{2}=-I d+\eta \otimes \xi, \quad g(\phi \cdot, \phi \cdot)=g-\eta \otimes \eta,
$$

where $\xi$ denotes the Reeb vector field of the contact structure, that is the unique vector field on $S$ such that

$$
i_{\xi} \eta=1, \quad i_{\xi} d \eta=0
$$

A contact metric manifold is said to be Sasakian if the following integrability condition is satisfied

$$
N_{\phi}(X, Y):=[\phi X, \phi Y]+\phi^{2}[X, Y]-\phi[X, \phi Y]-\phi[\phi X, Y]=-d \eta(X, Y) \xi,
$$

for any vector fields $X$ and $Y$ on $S$.

Two Sasakian manifolds $\left(S_{1}, \eta_{1}, g_{1}\right)$ and $\left(S_{2}, \eta_{2}, g_{2}\right)$ are said to be equivalent if there exists a contactomorphism $F: S_{1} \longrightarrow S_{2}$ between them which is also an isometry, i.e.

$$
F^{*} \eta_{2}=\eta_{1}, \quad F^{*} g_{2}=g_{1}
$$

One can prove that if (3) holds then $F$ satisfies also

$$
F_{*_{x}} \circ \phi_{1}=\phi_{2} \circ F_{*_{x}}, \quad F_{*_{x}} \xi_{1}=\xi_{2}
$$

for any $x \in S_{1}$. An isometric contactomorphism $F: S \longrightarrow S$ from a Sasakian manifold $(S, \eta, g)$ to itself will be called a Sasakian transformation of $(S, \eta, g)$.

It is a well-known fact [4] that the foliation defined by the Reeb vector field of a Sasakian manifold $S$ has a transversal Kähler structure. Using the theory of Riemannian 
submersions one can prove that the transverse geometry is Kähler-Einstein if and only if the Ricci tensor of $S$ satisfies the following equality

$$
\text { Ric }=\lambda g+\nu \eta \otimes \eta
$$

for some constants $\lambda$ and $\nu$. Any Sasakian manifold satisfying (4) is said to be $\eta$-Einstein (see [5] for more details).

A remarkable property of $\eta$-Einstein Sasakian manifolds is that, contrary to SasakiEinstein ones, they are preserved by $\mathcal{D}_{a}$-homothetic deformations, that is the change of structure tensors of the form

$$
\phi_{a}:=\phi, \quad \xi_{a}:=\frac{1}{a} \xi, \quad \eta_{a}:=a \eta, \quad g_{a}:=a g+a(a-1) \eta \otimes \eta
$$

where $a>0$.

By a Sasakian immersion (often called invariant submanifolds or Sasakian submanifolds in the literature) of a Sasakian manifold $\left(S_{1}, \eta_{1}, g_{1}\right)$ into the Sasakian manifold $\left(S_{2}, \eta_{2}, g_{2}\right)$ we mean an isometric immersion $\varphi:\left(S_{1}, g_{1}\right) \longrightarrow\left(S_{2}, g_{2}\right)$ that preserves the Sasakian structures, i.e. such that

$$
\begin{gathered}
\varphi^{*} g_{2}=g_{1}, \quad \varphi^{*} \eta_{2}=\eta_{1}, \\
\varphi_{*} \xi_{1}=\xi_{2}, \quad \varphi_{*} \circ \phi_{1}=\phi_{2} \circ \varphi_{*} .
\end{gathered}
$$

We refer the reader to the standard references $[2,4]$ for a more detailed account of Riemannian contact geometry and Sasakian manifolds.

Sasakian space forms. Recall that the curvature tensor of a Sasakian manifold is completely determined [2] by its $\phi$-sectional curvature, that is the sectional curvature of plane sections of the type $(X, \phi X)$, for $X$ a unit vector field orthogonal to the Reeb vector field.

A Sasakian space form is a connected, complete Sasakian manifold with constant $\phi$ sectional curvature. According to Tanno [20] there are exactly three simply connected Sasakian space forms depending on the value $c$ of the $\phi$-sectional curvature: the standard Sasakian sphere up $\mathcal{D}_{a}$-homothetic deformation if $c>-3$, the Heisenberg group $\mathbb{C}^{n} \times \mathbb{R}$ if $c=-3$ and the hyperbolic Sasakian space form $\mathbb{B}^{n} \times \mathbb{R}$ if $c<-3$. Notice that each simply connected space form admits a fibration over a Kähler manifold and in the non-compact cases the fibration is trivial.

We denote by $M(n, c)$ the simply connected $(2 n+1)$-dimensional Sasakian space form with $\phi$-sectional curvature equal to $c$. Every connected, complete Sasakian space form is Sasakian equivalent to $M(n, c) / \Gamma$, where $\Gamma$ is a discrete subgroup of the Sasakian transformation group of $M(n, c)$.

Immersions and regular foliations. We recall some basic concepts from foliation theory (see e.g. $[16,18]$ ). Let $M$ be a smooth manifold of dimension $n$. A foliation can be defined as a maximal foliation atlas on $M$, where a foliation atlas of codimension $q$ on $M$ is an atlas

$$
\left\{\varphi_{i}: U_{i} \longrightarrow \mathbb{R}^{n}=\mathbb{R}^{p} \times \mathbb{R}^{q}\right\}_{i \in I}
$$

of $M$ such that the change of charts diffeomorphisms $\varphi_{i j}$ locally takes the form

$$
\varphi_{i j}(x, y)=\left(g_{i j}(x, y), h_{i j}(y)\right) .
$$

Each foliated chart is divided into plaques, the connected components of

$$
\varphi_{i}^{-1}\left(\mathbb{R}^{p} \times\{y\}\right)
$$

where $y \in \mathbb{R}^{q}$, and the changes of chart diffeomorphism preserve this division. 
Definition 2. A foliated map is a map $f:(M, \mathcal{F}) \longrightarrow\left(M^{\prime}, \mathcal{F}^{\prime}\right)$ between foliated manifolds which preserves the foliation structure, i.e. which maps leaves of $\mathcal{F}$ into leaves of $\mathcal{F}^{\prime}$.

Now, let $(M, \mathcal{F})$ and $\left(M^{\prime}, \mathcal{F}^{\prime}\right)$ be foliated manifolds and $f: M \longrightarrow M^{\prime}$ be an immersion. Moreover, assume that $f$ is a foliated map. Thus

$$
f_{*_{x}}(L(x)) \subset L^{\prime}(f(x))
$$

for each $x \in M$, where $L=T(\mathcal{F})$ and $L^{\prime}=T\left(\mathcal{F}^{\prime}\right)$. In particular, it follows that $\operatorname{dim}(\mathcal{F}) \leq$ $\operatorname{dim}\left(\mathcal{F}^{\prime}\right)$. The proof of the following proposition is quite standard and will be omitted:

Proposition 3. $(M, \mathcal{F})$ and $\left(M^{\prime}, \mathcal{F}^{\prime}\right)$ be foliated manifolds of dimension $n$ and $n^{\prime}$, respectively, and $f: M \longrightarrow M^{\prime}$ be a foliated immersion. Suppose that $\operatorname{dim}(\mathcal{F})=\operatorname{dim}\left(\mathcal{F}^{\prime}\right)$. Then for each $x \in M$ there are charts $\varphi: U \longrightarrow \mathbb{R}^{p} \times \mathbb{R}^{q}$ for $M$ about $x$ and $\varphi^{\prime}: U^{\prime} \longrightarrow \mathbb{R}^{p} \times \mathbb{R}^{q^{\prime}}$ for $M^{\prime}$ about $f(x)$ such that

(i) $\varphi(x)=(0, \ldots, 0) \in \mathbb{R}^{n}$

(ii) $\varphi^{\prime}(f(x))=(0, \ldots, 0) \in \mathbb{R}^{n^{\prime}}$

(iii) $F\left(x_{1}, \ldots, x_{n}\right)=\left(x_{1}, \ldots, x_{n}, 0, \ldots, 0\right)$, where $F:=\varphi^{\prime} \circ f \circ \varphi^{-1}$

(iv) $L(x)=\operatorname{span}\left\{\frac{\partial}{\partial x_{1}}(x), \ldots, \frac{\partial}{\partial x_{p}}(x)\right\}$

(v) $L^{\prime}(f(x))=\operatorname{span}\left\{\frac{\partial}{\partial x_{1}}(f(x)), \ldots, \frac{\partial}{\partial x_{p}}(f(x))\right\}$

where $p=\operatorname{dim}(\mathcal{F})=\operatorname{dim}\left(\mathcal{F}^{\prime}\right), q=n-p, q^{\prime}=n^{\prime}-p$.

Let $\mathcal{F}$ be a foliation on a manifold $M$ and let $L$ be a leaf of $\mathcal{F}$. It is well known that $L$ intersects at most a countable number of plaques in a foliated chart $U$. Now we give the following definition.

Definition $4([18])$. A foliation $\mathcal{F}$ is said to be regular if for any $x \in M$ there exists a foliated chart $U$ containing $x$ such that every leaf of $\mathcal{F}$ intersects at most one plaque of $U$.

The following proposition is a generalisation to the non-compact case and to immersions of [11, Proposition 3.1]:

Proposition 5. Let $(M, \mathcal{F})$ and $\left(M^{\prime}, \mathcal{F}^{\prime}\right)$ be foliated manifolds such that $\operatorname{dim}(\mathcal{F})=$ $\operatorname{dim}\left(\mathcal{F}^{\prime}\right)$. If there exists a foliated immersion $f:(M, \mathcal{F}) \longrightarrow\left(M^{\prime}, \mathcal{F}^{\prime}\right)$ and $\mathcal{F}^{\prime}$ is regular, then $\mathcal{F}$ is also regular.

Proof. Assume that $\mathcal{F}$ is not regular. Then there exists a point $x \in M$ and a leaf $L$ of $\mathcal{F}$ such that, for any foliated chart $U$ containing $x, L$ intersects more then one plaque in $U$. Let us consider the foliated charts $U$ and $U^{\prime}$, respectively about $x$ and $f(x)$, satisfying the properties stated in Proposition 3. Then there exist at least two plaques, say $P_{1}=\varphi^{-1}\left(\mathbb{R}^{p} \times\left\{\mathbf{y}_{1}\right\}\right)$ and $P_{1}=\varphi^{-1}\left(\mathbb{R}^{p} \times\left\{\mathbf{y}_{2}\right\}\right)$, such that

$$
L \cap P_{1} \neq \emptyset, \quad L \cap P_{2} \neq \emptyset,
$$

where $\mathbf{y}_{1}, \mathbf{y}_{2} \in \mathbb{R}^{q}$. Notice that, for each $i \in\{1,2\}, f\left(P_{i}\right)$ is a plaque of $\mathcal{F}^{\prime}$ in $U^{\prime}:=f(U)$. Indeed, using Proposition 3, we have $f\left(P_{i}\right)=f\left(\varphi^{-1}\left(\mathbb{R}^{p} \times\left\{\mathbf{y}_{i}\right\}\right)\right)=\varphi^{\prime-1}\left(F\left(\mathbb{R}^{p} \times\left\{\mathbf{y}_{i}\right\}\right)\right)=$ $\varphi^{\prime-1}\left(\mathbb{R}^{p} \times\left\{\left(\mathbf{y}_{i}, 0, \ldots, 0\right)\right\}\right)$. Now, since $f$ is a foliated map, $L^{\prime}=f(L)$ is a leaf of $\mathcal{F}^{\prime}$ and from (8) it follows that $L^{\prime} \cap f\left(P_{1}\right) \neq \emptyset$ and $L^{\prime} \cap f\left(P_{2}\right) \neq \emptyset$. But this contradicts the regularity of $\mathcal{F}^{\prime}$. 


\section{Classification}

In this Section we prove the main result of this paper, that is the classification of connected, regular $\eta$-Einstein Sasakian manifolds immersed into Sasakian space forms.

Proof of Theorem 1. Let $M(N, c)$ be one of the non-compact simply connected Sasakian space forms and $\pi^{\prime}: M(N, c) \rightarrow K^{\prime}$ the (trivial) fibration over its Kähler quotient. Recall that $K^{\prime}$ is either $\mathbb{C}^{N}$ with its flat Kähler metric or the hyperbolic Kähler space form $\mathbb{B}^{N}$.

Since $S$ is complete and regular, by [19] there exists a fibration $\pi: S \rightarrow K$, whose fibers are the leaves of the Reeb foliation of $S$. By assumption $S$ is Sasakian $\eta$-Einstein and then $K$ is necessarily Kähler-Einstein (see [9]). The fibration $\pi: S \rightarrow K$ is a Riemannian submersion and since $S$ is complete then $K$ also is (see $[17,9]$ ).

By assumption there exists an open neighbourhood $U_{p}$ of $p \in S$ and a Sasakian immersion $\varphi: U_{p} \rightarrow M(N, c)$. By Proposition 5 the submanifold $U_{p}$ is still regular. The restriction of $\pi$ to $U_{p}$ gives then a projection of the Sasakian $\eta$-Einstein manifold $U_{p}$ to the Kähler manifold $\pi\left(U_{p}\right) \subset K$.

The Sasakian immersion $\varphi: U_{p} \rightarrow M(N, c)$ covers a Kähler immersion $i(\varphi)($ see $[7,10])$ making the following diagram commutative:

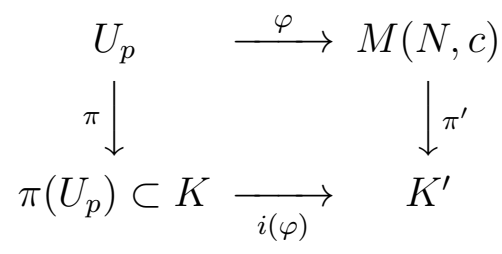

We have proved that there exists $q=\pi(p) \in K$, an open neighbourhood $V_{q}=\pi\left(U_{p}\right)$ of $q$ and a Kähler immersion of $V_{q}$ in $K^{\prime}$. By Umehara [21] $V_{q}$ is flat or complex hyperbolic.

On the other end, by [8] (see also [1, Theorem 5.26]), the Kähler-Einstein manifold $K$ is real analytic and by [6, Theorem 4 and Theorem 10$]$, for every $q \in K$ there exists an open neighbourhood $V_{q}^{\prime}$ and a Kähler immersion of $V_{q}^{\prime}$ in $K^{\prime}$. Then $K$ is locally flat and [9, Formula 1.31] implies that the $\phi$-sectional curvature of $S$ is less or equal to -3 .

By Tanno [20] there exists a discrete group $\Gamma$ of the Sasakian transformations of $S$ such that $S=M(n, c) / \Gamma$ and this proves the first part of the theorem.

For the second part of the theorem, let us suppose $U_{p}=S$. Reasoning as before, by completeness of $K$ and by Calabi's Rigidity Theorem [6] for Kähler immersions into Kähler space forms (see also [15]) one obtains the stronger result that either $K=\mathbb{C}^{n}$ or $K=\mathbb{B}^{n}$ and the projection is just the trivial fibration because in both cases $K$ contractible. Then, since $S$ is complete, the fibres of the fibration are diffeomorphic either to $\mathbb{R}$ or to $\mathbb{S}^{1}$.

The second case cannot occur because $\varphi$ is a Sasakian immersion and then it restricts to immersions on the leaves of the Reeb foliations of $S$ and $M(N, c)$. But the leaves of $M(N, c)$ are diffeomorphic to $\mathbb{R}$ and if the leaves of $S$ are circles we would obtain immersions of the circle in $\mathbb{R}$ which is not possible.

Now it remains to prove that $\varphi$ is the standard embedding up to Sasakian transformations.

First observe that, again by Calabi's Rigidity Theorem, the immersion $i(\varphi): K \rightarrow K^{\prime}$ has (up to unitary transformation) the following form:

$$
i(\varphi)\left(z_{1}, \ldots, z_{n}\right)=\left(z_{1}, \ldots, z_{n}, 0, \ldots, 0\right) .
$$

Because the fibrations are trivial $\varphi$ must have the following expression:

$$
\varphi\left(z_{1}, \ldots, z_{n}, t\right)=\left(z_{1}, \ldots, z_{n}, 0, \ldots, 0, f_{N}\left(\left(z_{1}, \ldots, z_{n}, t\right)\right)\right) .
$$


Since $\phi$ is a Sasakian immersion, in particular we have $\varphi^{*}\left(\eta_{N}\right)=\eta_{n}$, where $\eta_{N}$ and $\eta_{n}$ are the standard contact forms of $M(N, c)$ and $M(n, c)$ respectively. Then a direct calculation of $\varphi^{*}\left(\eta_{N}\right)=\eta_{n}$ yields $\frac{\partial f_{N}}{\partial t}=1$ and $\frac{\partial f_{N}}{\partial x_{i}}=\frac{\partial f_{N}}{\partial y_{i}}=0$ for $i=1, \ldots n$, where we put $z_{j}=x_{j}+i y_{j}$.

REMARK 6. In Theorem 1 the case $U_{p}=S$ cannot occur if $S$ is compact because if $S$ is compact, a Sasakian immersion cannot exist for otherwise, from the regularity of a compact Sasakian manifold, we would obtain a (compact) Kähler quotient immersed either in $\mathbb{C}^{N}$ or in $\mathbb{B}^{N}$, which is impossible by the Maximum Principle.

The following result is a variation of Theorem 1 :

TheOREM 7. Let $S$ be a $(2 n+1)$-dimensional connected, complete, $\eta$-Einstein Sasakian manifold. Suppose that for every $p \in S$ there exists an open neighbourhood $U_{p}$ of $p$ and a Sasakian immersion $\phi: U_{p} \rightarrow M(N, c)$, where $c \leq-3$. Then $S$ is Sasaki-equivalent to $M(n, c) / \Gamma$ where $\Gamma$ is some discrete subgroup of the Sasakian-isometry group of $M(n, c)$. Moreover, if $U_{p}=S$ then $\Gamma=\{1\}$ and $\phi$ is, up to a Sasakian transformation of $M(N, c)$, given by

$$
\phi(z, t)=(z, 0, t+c)
$$

Proof. For every point $p \in S$ we have an immersion of some $U_{p}$. After possibly shrinking the open set $U_{p}$ we obtain an open set where the Reeb foliation is given by a fibration over a Kähler base. Then we proceed exactly as in the proof of Theorem 1 and we obtain that $U_{p}$ (and then $S$ ) has constant $\phi$-sectional curvature at every point. Then $S$ is Sasakiequivalent to $M(n, c) / \Gamma$ where $\Gamma$ is some discrete subgroup of the Sasakian-isometry group of $M(n, c)$.

If $U_{p}=S$ we cannot directly conclude as in Theorem 1 because a priori we don't know if $M(n, c) / \Gamma$ is regular. On the other end we are assuming the existence of an immersion of $U_{p}=S$ into the regular Sasakian space form $M(N, c)$ and then $S$ is regular by Proposition 5. We can now apply Theorem 1 to conclude.

\section{REFERENCES}

[1] Besse, Arthur L., Einstein manifolds, Reprint of the 1987 edition. Classics in Mathematics. SpringerVerlag, Berlin, 2008

[2] D. E. Blair, Riemannian geometry of contact and symplectic manifolds, Birkhäuser, 2010

[3] W. M. Boothby, H. C. Wang, On contact manifolds, Ann. Math. 68 (1958), 721-734

[4] C. P. Boyer, K. Galicki, Sasakian geometry, Oxford Mathematical Monographs, Oxford University Press, Oxford, 2008

[5] C. Boyer, K. Galicki, P. Matzeu, $\eta$-Einstein Sasakian geometry, Comm. Math. Phys. 262 (2006), 177-208

[6] E. Calabi, Isometric Imbedding of Complex Manifolds, Ann. Math. 58 (1953), 1-23

[7] B. Cappelletti Montano, A. Loi, Einstein and $\eta$-Einstein Sasakian submanifolds in spheres, Ann. Mat. Pura Appl. (4) 198 (2019), no. 6, 2195-2205.

[8] D. M. DeTurck, J. L. Kazdan, Some regularity theorems in Riemannian geometry, Ann. Sci. École Norm. Sup. (4) 14 (1981), 249-260.

[9] M. Falcitelli, S. Ianuş, A.M. Pastore, Riemannian submersions and related topics, World Scientific Publishing Co., Inc., River Edge, NJ, 2004

[10] M. Harada, Sasakian space forms immersed in Sasakian space forms, Bull. Tokyo Gakugey Univ. Ser. IV 24 (1972), 7-11

[11] M. Harada, On Sasakian submanifolds, Tôhoku Math. J. 25 (1973), 103-109

[12] K. Kenmotsu, Invariant submanifolds in a Sasakian manifold, Tôhoku Math. J. 21 (1969), 495-500

[13] M. Kon, Invariant submanifolds of normal contact metric manifolds, Kōdai Math. Sem. Rep. 25 (1973), 330-336 
[14] M. Kon, Invariant submanifolds in Sasakian manifolds, Math. Ann. 219 (1976), 277-290

[15] A. Loi, M. Zedda, Kähler Immersions of Kähler Manifolds into Complex Space Forms, Lecture Notes of the Unione Matematica Italiana 23, Springer, 2018

[16] I. Moerdijk, J. Mrčun, Introduction to foliations and Lie groupoids, Cambridge University Press, 2003

[17] B. O'Neill, The fundamental equations of a submersion, Michigan Math. J. 13 (1966), 459-469

[18] R. S. Palais, A global formulation of the Lie theory of transformation groups, Mem. Amer. Math. Soc. No. $22(1957)$

[19] B. L. Reinhart, Foliated Manifolds with Bundle-Like Metrics, Ann. Math. 69 (1959), 119-132

[20] S. Tanno, Sasakian manifolds with constant $\phi$-holomorphic sectional curvature, Tôhoku Math. J. 21 (1969), 501-507

[21] M. Umehara, Einstein-Kähler submanifolds of complex linear or hyperbolic space, Tôhoku Math. J. 39 (1987), 385-389

Gianluca Bande, Dipartimento di Matematica e Informatica, Università di Cagliari, ITALY.

Email address: gbande@unica.it

Beniamino Cappelletti-Montano, Dipartimento di Matematica e Informatica, UniverSitÀ Di CAGLiari, Italy.

Email address: b.cappellettimontano@unica.it

Andrea loi, Dipartimento di Matematica e Informatica, Università di Cagliari, Italy. Email address: loi@unica.it 